\title{
Unified Fault, Resource Management and Control in ATM-based IBCN
}

\section{S. Sartzetakis}

Institute of Computer Science, Foundation of Research and

Technology - Hellas, Heraklion, GR71110, Crete, Greece tel: +3081

391727,fax:+3081 391601,e-mail: stelios@ics.forth.gr

P. Georgatsos

Algosystems S.A., 4 Sardeon St., N. Smyrni, 17121 Athens, Greece tel: +301 9310281, fax:+301 9352873, email:pgeorgat@algo.com.gr

\section{G. Konstantoulakis}

NTUA, Computer Science Department, Telecommunications Lab, Heroon Polytechniou 9, 15773 Athens, Greece tel: +301 7721494 fax: +301772 2530,email gkonst@telecom.ntua.gr

\section{G. Pavlou, D. P. Griffin}

Department of Computer Science, University College London, Gower Street, London WC1E 6BT, UK. tel: +44171 419 3687, fax: +44 171 387 1397, email: \{G.Pavlou, D.Griffin\}@cs.ucl.ac.uk

\begin{abstract}
In this paper we present the initial specification of a system that covers both the control and management planes of network operation, with emphasis on fault, performance, and configuration management. The system incorporates rapid and reliable ATM network layer self-healing mechanisms, intelligent load balancing, dynamic routing and resource management functions all interworking together with the overall goal to ensure cost-effective network performance and availability under normal and fault situations. Restoration mechanisms in the ATM network layer are integrated with the control and management plane functionality, aiming at providing an integral and network-wide treatment to the problem of fault recovery. The system is designed and is being implemented adopting the emerging TINA framework and using a CORBA-based distributed processing environment. The validity and effectiveness of the system are assessed and demonstrated through international ATM field trials.
\end{abstract}

\section{Keywords}

Integrated ATM control and management. Fault management and network survivability. Dynamic routing, self-healing, OAM. TINA-C, CORBA DPE. 


\section{INTRODUCTION}

In order to minimise the cost of building and maintaining wide area ATM networks, efficient and cost-effective resource management techniques are needed to avoid the over-allocation of resources. This requires building a network that has the intelligence to monitor, control, and enforce its own QoS agreements, guaranteeing the high availability expected without the unnecessary cost of over-resourcing

Research in resource and routing management has led to a number of optimising algorithms, tackling the problem of network availability and performance in isolation, not considering the interactions between the control and management functions. It does not take explicitly into account the diversity of performance and bandwidth requirements of the many service classes supported by the networks. Furthermore, the work to date does not take an integrated approach to resource control, routing and alarm management from the perspective of network reliability and availability.

Recognising the need for combining the functional capabilities of the control and management planes for the benefit of network operation, this paper introduces the REFORM (REsource Failure and restORation Management in ATM-based IBCN) (REFORM, 1997) system with the purpose to ensure network performance and availability within acceptable levels under normal and fault conditions. The specifications of this system both from the functional and architectural aspects is the main theme of this paper.

Our system integrates control and management plane functionality for providing a complete, network-wide solution to the problem of network availability under normal and fault conditions. Specifically, control plane functions such as route selection, Operation Administration and Maintenance (OAM), and self-healing mechanisms are integrated with higher level network-wide routing and resource management functions.

We treat the problem of network availability in ATM-based IBC networks in an integral manner. The term "integral" refers to the ability to:

- cover the complete failure management cycle, that is prior, post failure, and failure normalisation phases;

- involve both control and management plane functionality.

While research has already been conducted in the area, it is the first time that a complete system is introduced that provides:

- an integrated approach to the problem of network performance and availability, considering the whole failure management cycle and integrating the OAM and control mechanisms with the network management functions in the performance and fault management areas;

- intelligent load balancing, OAM restoration, dynamic routing and spare resource management mechanisms all interworking together, explicitly taking into account the multi-service nature of the network environment.

The REFORM system has been designed according to the TINA architectural framework, using a CORBA-based distributed processing environment with all the advantages this brings and which are explained later in this paper. While the distinction between the control and management planes is maintained at a conceptual level, this distinction is relaxed during the system design and implementation phases. Part of control and management plane functionality will be modelled and implemented based on a DPE platform, using a uniform set of DPE services. The REFORM system is one of the first systems to provide such integrated solutions. In the following section we provide briefly some background information we consider helpful in understanding the scope of our system. Section 3 presents the REFORM system specifications, and section 4 presents the REFORM architecture. Following that, we describe a realistic environment scenario that is used to assess the functionality and performance of the 
REFORM system as whole. Finally Section 6 summarises the paper and describes aspects of future work.

\section{BACKGROUND INFORMATION, STATE-OF-THE-ART}

Considering networks as multiple-resource, multiple access systems, an obvious issue is how access to the resources is granted. Resource management refers to the necessary means and functions for resolving such contention problems with the overall objective to obtain solutions satisfying certain cost-effective criteria but constrained by general performance requirements. ATM networks by their very asynchronous multiplexing nature and the flexibility of the VPC layer that they offer, provide several degrees of freedom in traffic multiplexing. Although this flexibility eases the task of traffic admission and multiplexing it may have a disastrous effect on network performance if it is not managed properly. The problems and indeed the solutions of resource management are related to those of routing management - routes in ATM networks are defined in terms of VPCs and VPCs are defined in order to support routing.

Routing functionality is spread over the control and management planes of the network and the network elements. Route selection is part of the call control functions running at the network switches, whereas route definition functionality belongs to the management plane or it could itself provided by the control plane, on the basis of suitable distributed routing protocols (e.g. PNNI). Different sets of route may be defined for different connection types according to their performance requirements.

Successful fault management requires that information is gathered from network elements in a standardised way. Practical experience on existing networks shows that some fault scenarios involve alarm burst phenomena (e.g. uncontrolled pouring-in of numerous redundant alarm notifications) which tend to overload the management system.

Network robustness can be achieved with the deployment of autonomously operating restoration mechanisms. These restoration mechanisms reside at different network levels and different sub-networks and inter-work with each other through escalation schemes. The autonomously operating restoration mechanisms, provided by the network need to interact with the network management plane, in order to achieve the goal of network robustness. The network management system can simply monitor the restoration process or it can also actively participate in this process in order to complement and optimise the effect and operation of the self-healing actions taken at the control plane. Fast control plane restoration (self-healing) mechanisms as well as medium or longer term restoration mechanisms, in the management plane must be accommodated.

There are two possible self-healing techniques that can be used for restoration management. The first is the pre-assignment of VPCs, that are characterised as back-up VPCs; the second is a flooding based dynamic route search scheme. The pre-assignment of the back up VPCs yields several benefits compared to the flooding based dynamic route search scheme used in most existing self healing schemes. Its primary advantages are restoration rapidity and realisation of path restoration between path termination nodes. In this scheme there is the need for the management of back up VPCs as well as of original VPCs. In particular this restoration mechanism requires a message transmission system which may be based on OAM flows or other standardised protocols (e.g. PNNI), a spare resource management algorithm which is based on iterative algorithms in order to achieve optimum utilisation of network resources and also a well defined failure management cycle which can be realised as components of a management system.

The ATM-based networks operation and maintenance is organised in a layered fashion. Five hierarchical OAM layers and the relative OAM information flows have been defined (F5-1: Virtual Channel, Virtual Path, Transmission Path, Digital Section, Regenerator Section). The 
two first OAM layers (F4-F5) are in the ATM layer and the rest three (F1-F3) in the physical layer. The importance of the integration between management system and OAM has been identified by ITU (1992). Recommendation I.610 proposes an interaction scheme between the TMN and OAM. This scheme involves both $\mathrm{X} \& \mathrm{Q}$ interfaces for the entire path starting from the customer premises, passing the access local exchange up to the core network.

\section{REFORM SYSTEM SCOPE, APPROACH, AND FUNCTIONAL SPECIFICATIONS}

Adopting a network operator's viewpoint and by taking into account the broadband and multiservice nature of IBC network environment, the scope of the REFORM system is to provide the necessary means and functions for ensuring network performance and availability within acceptable levels under normal and fault situations. Ensuring network performance means the network will be able to maintain the performance of the existing connections within acceptable levels. Ensuring network availability means the network will be able to set-up connections guaranteeing their performance requirements. To achieve this, the REFORM system offers:

- fast fault detection, alarm indication and report functions;

- rapid and reliable network self-healing mechanisms for resource and service restoration, spread across both the control and management planes of the network operation;

- efficient dynamic and/or static resource and routing management schemes and algorithms with inherent load balancing functionality, coping with fault conditions; and

- efficient resource migration algorithms.

All these will interwork together with the overall goal to ensure the:

- cost-effective network performance and availability in normal conditions; and

- cost-effective, reliable and robust network recovery in the performance of existing connections as well as of the availability for new connections, from fault situations.

The term self-healing, as used above, refers to the ability of the network to reconfigure itself around failures quickly and gracefully with the goal of restoring service availability within acceptable levels for existing as well as future connections. Self-healing implies resource restoration by means of a distributed mechanism, as opposed to centralised schemes. Selfhealing mechanisms mainly reside in the control plane (one node), while higher level decisions can be escalated to the management plane. The REFORM system provides self-healing mechanisms at both the control and management planes. The latter is provided as part of suitable distributed routing and resource management schemes, dynamically adapting to fluctuating traffic load and in cases of resource failure. Centralised protection is also provided through management activities for defining suitable sets of routes and for allocating spare resources, in a cost-effective manner. These activities are adaptive in a quasi-static form regarding network resource failures, redesigning new sets of routes or reassigning spare resources. When failures occur and the lower-level self-healing capabilities of the network cannot succeed in restoring network availability under the current traffic conditions these activities may also be triggered. The REFORM system integrates all these functions through a carefully designed hierarchical architecture. Aspects of this architecture are discussed in Section 4.

To facilitate hierarchical designs and functional decomposition, we adopt the distinction between the management and control planes in the operation of networks. Such a distinction is necessary since it maps to the different domains and actors' interfaces currently found in the telecommunications arena; those of network equipment manufacturers, network operators and management system providers. In general, the control plane is required for the operation of the network, including the base functionality of signalling, Call Control (CC), and Operation 
Administration and Maintenance (OAM). The management plane is required for the optimal operation of the control plane, by tuning and appropriately managing various operational parameters in the control plane.

With respect to fault management, the approach adopted is that the control plane encompasses the functionality of fault detection and fast-responding self-healing mechanisms through the OAM capabilities that it offers. The management plane encompasses resource and routing management functionality for tuning and optimising the operation and effect of the control plane actions as well as for guaranteeing cost-effective allocation and usage of the spare resources in the network. Re-routing is also provided for restoring network availability to future connections. It is performed through a mixture of distributed and centralised logic, with the purpose to avoid damaged network areas while at the same time relieving the load from the back-up network resources. If such activities fail to restore network performance and availability given the current traffic conditions, other management activities will be activated (e.g. for defining new sets of routes, service migration). While the control plane functionality provides a fast reaction to a fault situation, the management plane provides the required short, medium or longer term reactions, depending on the severity of the failure and the actual traffic conditions, complementing the effect of the self-healing actions taken at the control plane. We broaden therefore the scope of restoration management beyond the level of self-healing mechanisms for service restoration at a local level. By incorporating effective resource and routing management functionality -suitably coping with fault conditions- our system restores service availability for existing and new connections, at a network-wide level. We build upon the results of the RACE II projects ICM (1997), IMMUNE (Nederlof, 1995), and TRIBUNE, and on other proven results in the literature (Fujii, 1994), (Griffin, 1995).

\subsection{System Functional Specifications}

We are concerned with failures caused in or escalated to the ATM network layer. For example, when a physical link failure has occurred and cannot be recovered by the restoration mechanisms in the physical layer(s), the fault is escalated to the ATM layer. This type of failure will cause a large number of alarms, as many as the VPLs defined on this link. REFORM therefore will provide for alarm correlation functionality in order to determine the original link failure. Faults in the ATM layer are also concerned with equipment related failures e.g. VP cross-connect, VPC TP (Termination Point) failures. The failures will be identified through the standardised OAM alarms. Restoration mechanisms and escalation procedures in the network levels below the ATM layer are not addressed.

The REFORM system incorporates rapid and reliable ATM layer self-healing mechanisms, intelligent load balancing, dynamic routing and resource management functions all interworking together with the overall goal to ensure cost-effective network performance and availability under normal and fault situations. Cost-effective solutions are pursued, on one hand by means of considering suitable objective functions in the optimisation problems corresponding to the route definition, spare resource allocation and management algorithms, and on the other hand by considering different survivability service classes.

In the rest of the section we outline the scope of the functionality of our system per each of the network phases with respect to failures. We concentrate on describing how control and management functionality is integrated to fulfil the objectives of our system. 


\section{Cost-effective Performance \& Availability under Normal \& Fault Conditions}

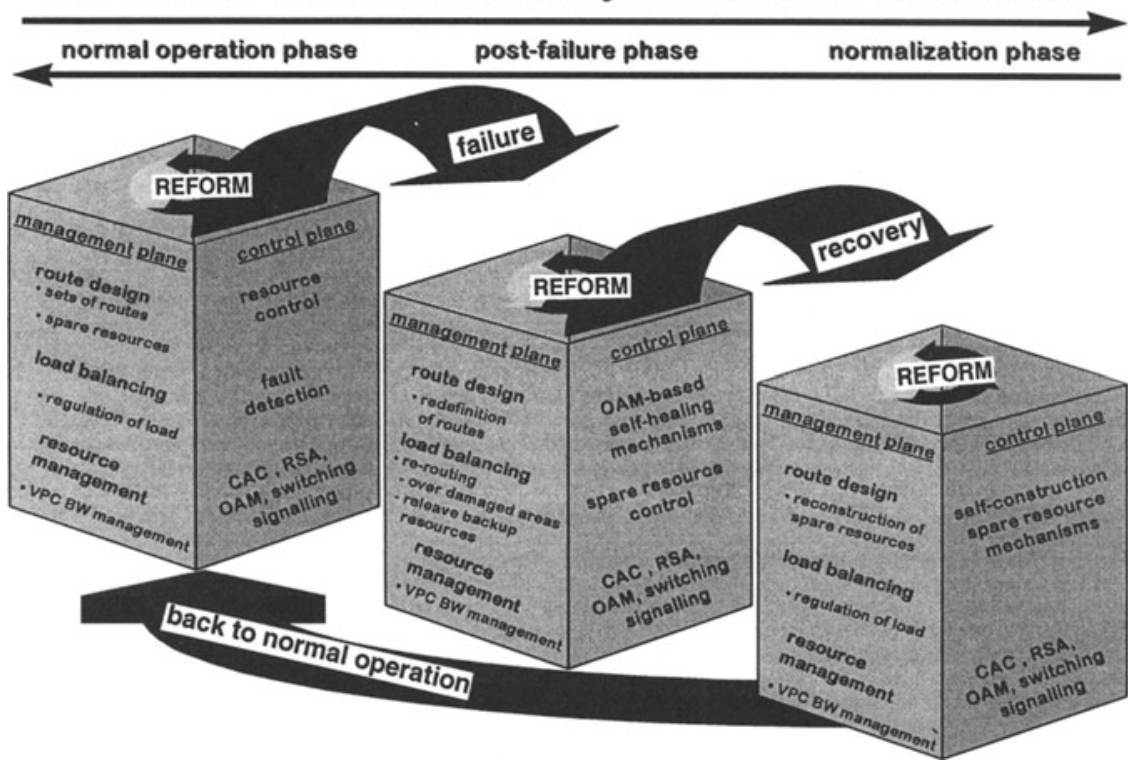

Figure 1 The REFORM system during the network operation phases.

During normal operation of the network, the system incorporates intelligent routing, load balancing and resource management functions, which tune the operation of the control plane through appropriate management actions. All this functionality is provided through a suitable hierarchy corresponding to different levels of abstraction and time-scales. The overall aim during this phase is to guarantee effective utilisation of network resources and cost-effective network availability within acceptable levels, while ensuring that the performance requirements of the various service classes are met. The route design functions are responsible for the design of appropriate admissible sets of routes per connection type, based on previously defined sets of VPCs, taking into account the performance requirements of the different connection types that the network supports. The route design functions are also responsible for the allocation of appropriate spare resources in the network according to certain cost-effective objectives and an overall protection strategy to provide as many options as possible for bypassing potential faults. Additionally, they should provision for adaptability to cater for unpredicted traffic fluctuations but according to the constraints of costeffectiveness. The load balancing functions (which may distributed or centralised) operate on the defined sets of routes trying to influence the routing decisions taken in the network switches, by conveying to them network-wide information. Through their activities load distribution may be regulated over the whole network. Note that balanced networks have the potential benefit of minimising disruptions of existing connections in case of network eventualities (node, link failures). The control plane encompasses the base functionality of CC, including routing and $\mathrm{CAC}$, signalling and $\mathrm{OAM}$. The routing functionality in the control plane is responsible for taking the actual routing decisions by means of suitable route selection algorithms. The control plane routing algorithms may also perform load balancing at a local level (i.e. at the vicinity of a node). The resource management functions are responsible for the management of the bandwidth of the VPCs according to actual network conditions. The system employs such functions both in the control and management plane, differing in terms 
of time-scale and abstractions. They try to ensure effective usage of VPCs and to avoid congestion at the cell and the connection level.

During the post-failure phase the system employs fast-responding self-healing functions in the control plane through the OAM facilities that it offers. The system follows a self-healing scheme based on pre-assigned back-up resources, rather than based on flooding messages. The standardised OAM flows are used to provide a fast and reliable means for inter-node message exchange. In addition, the adaptive, distributed and/or centralised routing, load balancing and resource management functionality of the system, with their inherent self-healing capabilities, will be activated for tuning the operation and complementing the effect of the control plane actions, to a network level. The overall goal during this phase, is to ensure a rapid recovery of network performance and availability, taken into account the actual traffic conditions. The entire system functionality is properly integrated through the levels of the REFORM hierarchy. The system combines the merits of distributed and centralised control schemes during the restoration process. Once a failure has been detected, the control plane will react through its OAM-based self-healing procedures and the associated automatic re-routing mechanisms. Spare resource control is also required for resolving competition as spare resources are normally shared. The load balancing and resource management activities in the management plane will also be in effect, through their own self-healing capabilities for providing a first level network-wide treatment to the restoration procedure. The load balancing functions will influence routing decisions so that future connections avoid routes traversing damaged paths, while at the same time taking care not to overload the back-up routes. The resource management activities will try to ensure that sufficient bandwidth on the back-up resources can be allocated. All these activities will take a network-wide view and the actual network traffic conditions. The system monitors network performance and availability with a wide perspective during the restoration process, in order to assess the effectiveness of the actions taken so far. If network performance is found unacceptable, the centralised routing functions will be activated for assigning new sets of routes and VPCs as necessary. The system allows for different survivability classes for prioritising access to network resources. If required, the restoration process may also be extended to higher levels for performing appropriate service migration functions, an extension of the system currently under study.

The normalisation phase is concerned with the reconstruction of routes and spare resources to cater for the predicted traffic and to build in flexibility to allow a rapid response to subsequent failures. The overall goal is to enter the normal operation mode by causing the minimum of disruptions to existing connections. The route design functions will determine new sets of routes and spare resources according to a general strategy satisfying certain costeffective criteria and taking into account network usage predictions. Different policies for spare resource reconstruction, like for example distributed self-reconstruction mechanisms are under investigation. The load balancing and resource management functions operate as in the normal operation case taking into account the newly resulted configuration. The control plane $\mathrm{OAM}$ and routing functionality will also be activated according to the reconstruction directives given by the management plane.

The detailed description of a functional model decomposing the above functionality into individual components and specifying the information exchanges between them is beyond the scope of this paper.

\section{SYSTEM ARCHITECTURE}

The key aspect of the REFORM architectural approach is the adoption of the emerging TINA framework with the purpose of evaluating its suitability and assessing its potential benefits when applied to network management, while comparing it as well to established technologies 
(OSI-based TMN, Internet SNMP). We use a commercial CORBA-based platform for providing the Distributed Processing Environment (DPE) for designing and implementing both the control and management plane functionality. Existing TINA-C architectures in the area of Resource Management are taken into account. Also existing TMN architectures for configuration and performance management, such as the ICM Virtual Path Connection and Routing Management (Griffin, 1995), (ICM, 1997), constitute the starting point for the management plane architecture; these are enhanced and modified in the light of the TINA-C developments and ported on the CORBA-based DPE. They are complemented by new functionality, not yet addressed by TINA and not available in existing TMN architectures.

Management Plane

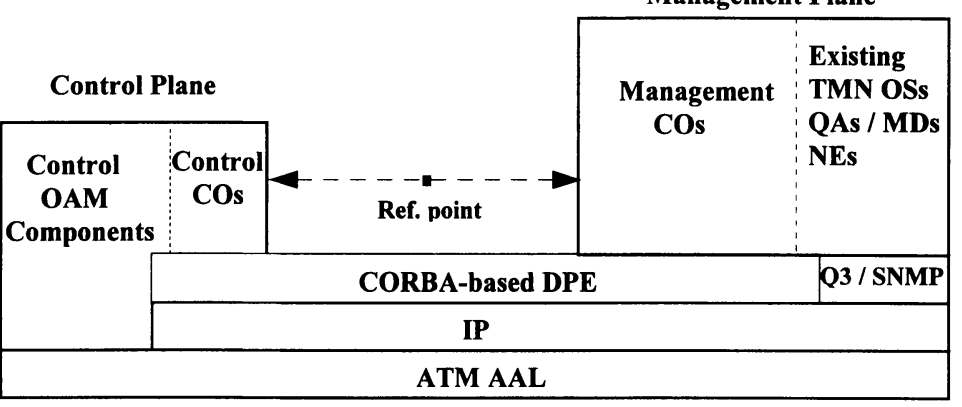

Figure 2 Overall REFORM system architecture.

The overall system architecture is shown in Figure 2. There exist two major domains, related to management and control plane respectively. The major underlying assumption is the use of TINA-C engineering principles in the form of a CORBA-based DPE.

The detailed architecture of the management plane takes into account state of the art work in this area i.e. in TINA-C and the RACE-II ICM project. The management plane functionality is specified as Computational Objects (COs) for Fault, Performance, Connection and Resource Configuration Management. Existing TMN applications from previous work - Operations Systems (OSs), Q-Adaptors (QAs) - are integrated in the REFORM system. Also, Network Elements (NEs) are accessed through TMN Q3 or SNMP interfaces. The integration of those elements and applications in the TINA-based REFORM environment raises issues of migration from TMN to TINA, which are discussed below. The architecture of the management domain has both the hierarchical aspects, as dictated by the TMN layered architecture which is adopted by TINA, and also the flat peer-to-peer aspects which are facilitated by the use of a DPE-based computational decomposition. Federation of activity between management domains is also considered.

The control plane functionality will be supported by relevant control logic and OAM transport protocols operating directly over the ATM adaptation layer. The state of the art in terms of projects such as IMMUNE and TRIBUNE together with the relevant ongoing ITU-T work is the starting point. The integration between the control and management plane domains will be accomplished through the interaction of computational objects over the DPE. As such, the control plane domain includes computational objects for the purpose of interfacing to the management plane. Such interactions are expected to be bi-directional.

All functions and operational components of our system in the leftmost box in Figure 2 are regarded as local inside a node, and will be implemented according to the particular capabilities of the network elements. We take into account the low level interfaces that provide connection with the hardware subsystem. Thus, direct message exchange will be feasible.

Overall, there are three types of transport in the REFORM system: 
- the ATM AAL, which supports the control plane interactions; the direct use of the ATM AAL ensures high-speed interactions but the relevant presentation and distribution facilities are minimal;

- the TMN Q3 using RFC1006/TCP/IP over the ATM AAL; this supports management plane interactions for the existing TMN applications; and

- the CORBA-based DPE using TCP/IP over the ATM ALL; this supports the majority of the management plane interactions and the interactions between the control and management domains.

There is also a possibility to use the CORBA-based DPE directly over the ATM AAL. This depends on developments on CORBA mappings in the lifetime of REFORM.

In order to be able to test our system under stress and worst case conditions all OAM protocols are integrated within the testing systems we use for our experimentation. The test tool is attached to the switches and monitors the data streams, OAM flows and collects statistical information. On the other hand we are able to generate fault in the OAM flows and collect statistical information. We are also able to generate faults in the OAM streams in order to cause predefined error profiles.

\section{Migration from TMN to TINA}

REFORM tackles aspects such as how to integrate or migrate towards the TINA environment, using in particular the DPE engineering model and the problem decomposition from the enterprise, information and computational viewpoints.

Given the fact that network elements will support TMN Q3 or SNMP management interfaces while existing TMN management applications (OSs) with Q interfaces may be reused, issues in migrating these components to the TINA environment will have to be tackled. In addition, existing TMN architectures for ATM resource management (e.g. ICM) will be reworked according to the new methodologies and the architectural input from TINA-C in these areas.

In the key area of integrating elements or applications with Q3 interfaces, the X/Open Joint Inter-Domain Management (XoJIDM, 1996) task force has been investigating the similarities and differences between GDMO/ASN.1 and CORBA IDL object models and proposes generic translations in both directions. It should be mentioned that elements with SNMP interfaces may also be seen as Q3-capable elements through generic translation: the ICM project has contributed to the relevant NMF specifications and has developed a generic Internet $\mathrm{Q}$ Adaptor (IQA) which is available to REFORM.

Given the fact that fault and performance management need fine-grain event reporting/logging and sophisticated query and retrieval facilities, REFORM will assess the suitability of OMG Common Object Services (COS) to support such functionality. Specialised facilities in addition to the OMG common services will also be investigated, such as management brokers that enable multiple object access and sophisticated information retrieval, sophisticated event reporting and logging facilities, metric monitoring and summarisation facilities etc. In general, the full expressive power of the OSI Management / TMN framework needs to be available over the CORBA-based DPE.

\section{AN EXPERIMENTATION SCENARIO}

Our work is centred around a number of trials and experiments to validate the system in a realistic environment. The field trials are based on European National Host (NH) platforms (ACTS, 1997). The NHs are consolidated advanced platforms comprising communications infrastructure, services and generic applications. The NHs are made available to projects interested in performing operational trials or experiments of leading edge applications, services and management that involve real networks, services and users. 
As an example of a typical, experimental scenario, the following describes a fault in the delivery of video services and the associated activity of the REFORM system to resolve the problem. Consider a video distribution service consisting of a video server which distributes a video stream to the users of the service on demand. (Figure 3). Faults can affect this service in a number of ways. For example: a user may be unable to access the video distribution server to request a new video stream; an existing video stream may be interrupted; or the video server itself may collapse. In the following a fault caused by a link failure is discussed.

On failure there are a number of ways of restoring the service. These can be at many levels of the REFORM architecture: from the reception of OAM alarms and the invocation of fast, localised self-healing mechanisms through to network wide, off-line redesign of the network routes available to the video services. The goal of our system is to enable these mechanisms to work together in an integrated way. By the co-operation of algorithms and functions in each of the architectural levels the network can be made operational as quickly as possible through the immediate response of localised algorithms embedded in the network elements while the higher level management algorithms restore efficiency in the network as a whole over a longer time period.

Figure 3 shows a simplified view of a sample network configuration formed by the EXPERT testbed (involving the Swiss NH and the Dutch $\mathrm{NH}$ ) and the Norwegian NH testbed. A video distribution server is attached to the LATEX node of the Swiss $\mathrm{NH}$, there is a single user group attached to the NT2 node of the same network and a second user group located in the Dutch NH.

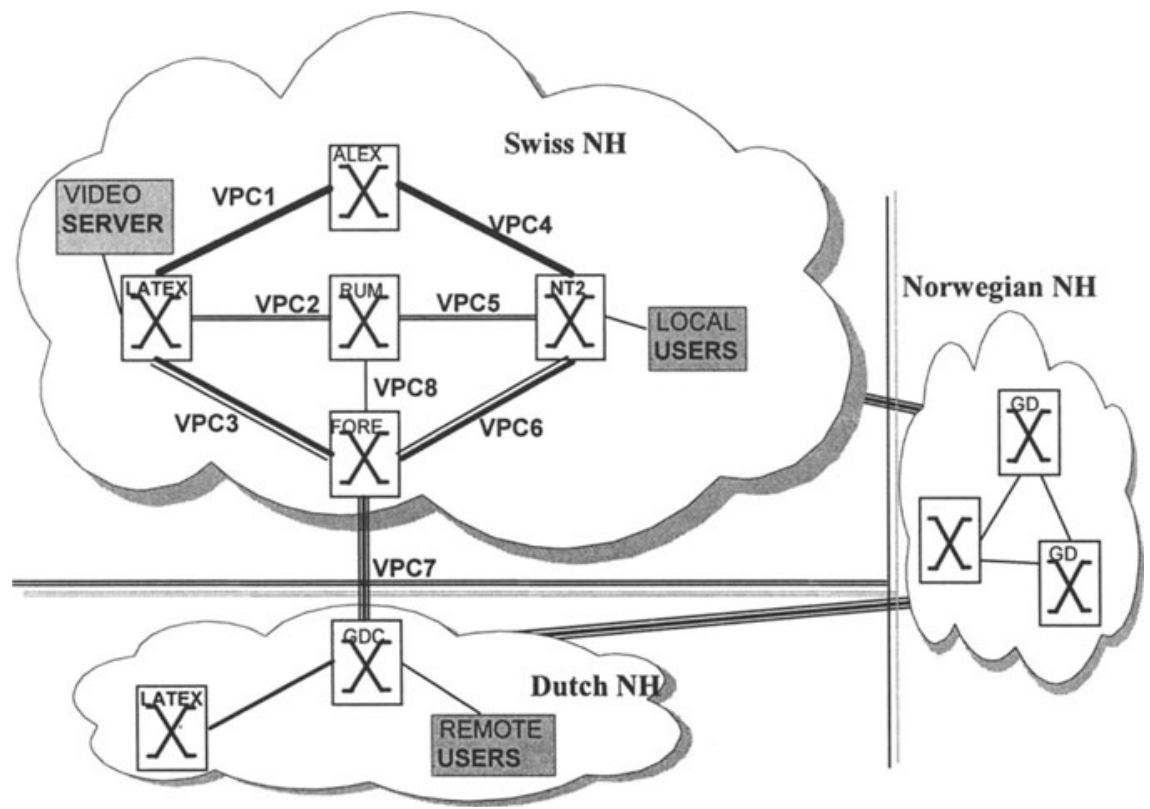

Figure 3 The NH set-up for the REFORM experiments.

Video streams are transmitted via VCCs which are set up on demand between the users and the server and routed over previously installed VPCs. In this example, two possible routes have been assigned to video traffic between the video server and the local users on the Swiss NH: over VPC1 and VPC4; or over VPC2 and VPC5. Similarly, two routes have been 
assigned to the remote users at the Dutch NH: over VPC3 and VPC 7; and over VPC2, VPC8 and VPC7.

The fact that two routes have been assigned for each source-destination pair is implemented in the network by alternative route selection entries in the VC switches of the network nodes. When a connection request is made, Route Selection Algorithms (RSAs) determine which route should be used (Georgatsos, 1996). The route selection entries are defined initially by the route design algorithms in the upper levels of the REFORM architecture (note that this is an example of the co-operation between the management and control levels of the REFORM architecture).

During normal operation of the network, the RSAs select appropriate routes for new connections according to their algorithms (Georgatsos, 1996). The load balancing functions manage the route selection criteria used by the RSAs to balance the load over the network as evenly as possible by altering the selection priorities associated with each route selection entry.

In this scenario we now introduce a fault in the link between the LATEX and ALEX nodes in the Swiss NH, so putting VPCl out of service and disrupting the video streams which were allocated to VCCs routed over this VPC.

On failure of this link, the LATEX node is notified by a control plane signal (OAM alarm) that the link is out of service. On receipt of this signal, OAM-based restoration protocols will attempt to restore the failed link. Because this may take a large amount of time in the case of physical failures requiring human intervention, the local control plane self-healing algorithms will prohibit the RSA from selecting this VPC for future connections. As a secondary response the control plane algorithms will try to obtain as much bandwidth as possible for the alternative route (VPC2 and VPC5) from the common pool bandwidth which is continually updated by the higher level bandwidth management functions which redistribute capacity to existing routes and VPCs according to measured and predicted traffic levels.

As new connections are established they are routed over the only remaining route and it is likely that it will become overloaded leading to connection rejections. Before this situation arises, the Load Balancing component, in the management plane, will register a high traffic load compared to the network wide average and attempt to resolve this problem. However, there are no alternative routes available from the LATEX node, and Load Balancing is unable to resolve the potential problem.

At this point, Load Balancing informs its superior component in the REFORM management hierarchy, Route Design, of an unbalanced network which it is unable to resolve (Griffin, 1995). Route Design then attempts to redesign the VPC network and the routing plan to cope with the new physical topology. In this case potential new routes are considered (VPC2, VPC8 and VPC6; or VPC3 and VPC6) according to existing and predicted traffic and the quality constraints of the video service (for example there may be a maximum number of links and nodes video traffic may be routed over due to maximum allowable delay figures) and a new route is established if possible. The management decisions are implemented in the network by updating route selection tables in the RSAs at the corresponding nodes. As time progresses Load Balancing will update the route selection priorities for the new and the already existing VPCs, to balance the load in the overall network and in the control plane the RSAs will make all new routing decisions according to the new routing table entries.

The above is an outline of the functionality of the REFORM system highlighting the cooperation required in an integrated system covering the control and management planes. The overall problem of fault, resource management and control is handled by a number of layers. Each layer has a well defined limit of responsibility with operational parameters defined and managed by the layer above. 


\section{CONCLUSIONS, FUTURE WORK}

We introduced an integrated framework for ensuring network performance and availability in multi-class ATM networks under normal and fault conditions. We integrated control and management plane functionality, covering the complete failure management cycle (prior, post, normalisation). We combine in one system:

- rapid and reliable network self-healing mechanisms, spread across the control and management layers of the network operation;

- intelligent routing coping with fault conditions, taking into account the multi-service environment;

- intelligent load balancing functionality with inherent self-healing capabilities;

- cost-effective spare resource allocation and appropriate management schemes

We provide a number of generic and specific software components to offer solutions to the problems of fault, and routing, management for ATM networks. These components can be exploited by the existing network operators, or provided to new network operators as part of a packaged solution.

Our goal is also to make substantial contributions to TINA-C especially in the fields of fault and performance management as well as to the definition of the migration path from currently used management frameworks, like TMN, to emerging ones, like TINA. Since we adopted the emerging TINA framework based on a commercial CORBA-type platform, we will evaluate, validate and demonstrate through experimentation in real network environment the suitability of such approaches to network management.

We plan to carry out field trials promoting in parallel the use of advanced networks and services as they are provided by $\mathrm{NH}$ environment incorporating real user communities in Europe. This way we demonstrate how the functional capabilities of the developed system can improve performance and reliability of end-to-end connections.

Much of the above work is still on-going, and experimentation is planed for this and the next years. Up to date information is widely available on line (REFORM, 1997).

\section{Acknowledgements}

REFORM is a research project (July 96 - June 99) of the ACTS programme partially funded by the Commission of the European Union. The authors wish to thank all their project colleagues who contributed in many ways to the formation of the ideas described here. Without them this work and our co-operation for these three years would never have started.

\section{REFERENCES}

REFORM (1997) "REFORM System Requirements and Analysis", Deliverable D1, A208/Bell/WP1/DS/P/002/b1. On-line information on the REFORM system is available under: http://www.algo.com.gr/acts/reform

ACTS (1997) On-line infomation: http://www.uk.infowin.org/ACTS/

Fujii H. and Yoshikai N. (1994) "Restoration Message Transfer Mechanism and Restoration Characteristics of Double-Search Self-Healing ATM Network", IEEE Journal on Selected Areas in Communications, Vol.12, No.1.

Georgatsos P., Griffin D. (1996) "A Management System for Load Balancing through Adaptive Routing in Multi-Service ATM Networks", INFOCOM 1996.

Griffin D., Georgatsos P. (1995) “A TMN system for VPC and routing management in ATM networks", Integrated Network Management IV, Proc. of $4^{\text {th }}$. ISINM 1995, ed. A.S.Sethi, et al., Chapman \& Hall, UK, 1995. 
ICM (1997) "Integrated Communications Management of Broadband Networks", ed. Griffin D., Crete University Press, Heraklion, Greece, ISBN 9605240068.

ITU-T (1992) Recommendations: M.3010, Principles for a Telecommunications Management Network, Geneva, October 1992. / M.3200, TMN Management Services: Overview, Geneve, October 1992. I M.3100 Generic IM / I.610 / ITU-T SG4 "TMN Architecture and Principles, Management Services, Generic Object Model" / ITU-T SG11 "TMN Protocols and Interface Models"

Nederlof L., et al (1995), "End-to-End Survivable Broadband Networks" IEEE Communications Magazine, September 1995.

TINA-C (1994) Documents: Overall Principles and Concepts of TINA (1995), Fault Management and Resource Configuration Management, Connection Management Architecture, Management Architecture, Network Resource Information Model Specification.

XoJIDM (1996) (X/Open Joint Inter-Domain Management task force) NMF-X/Open; Proposal "InterDomain Management: Specification Translation" (from ASN.1 to IDL).

\section{BIOGRAPHIES}

Stelios Sartzetakis received his BSc degree in Mathematics from Aristotelian University of Thessaloniki in 1983, and his Masters in Systems and Computer Engineering from Carleton University of Ottawa, Canada in 1986. He joined ICS-FORTH in 1988 where he has been responsible for FORTH's telecommunications infrastructure at large, principal in the creation of FORTHnet, and participated in a number of EU funded projects. Today he is senior telecommunications engineer in the networks group (www.ics.forth.gr/ stelios), responsible for research projects in broadband telecommunications networks and services management.

Panos Georgatsos received the B.S. degree in Mathematics from the National University of Athens, Greece, in 1985, and the Ph.D. degree in Computer Science, with specialisation in network routing and performance analysis, from Bradford University, UK, in 1989. Dr. Georgatsos is working for Algosystems SA, Athens, Greece, as a network performance consultant. His research interests are in the areas of network and service management, analytical modelling, simulation and performance evaluation. $\mathrm{He}$ has been participating in a number of telecommunications projects within the framework of the EU funded RACE and ACTS programmes.

George Konstantoulakis was born in Chania, Greece at 1968. He received the Degree in Electrical Engineering from NTUA in 1992. In 1991 he joined the Telecommunications Laboratory of NTUA where he finished his thesis and now finishing his Ph.D. His research interests are in the area of broadband communication networks, high speed - real time architectures and algorithms. He has over ten publications in the above areas. Member of the IEEE and the Technical Chamber of Greece by which he took the reward of the new engineer with the best graduate thesis (1993) at national level. He is reviewer of IFIP TC6.

George Pavlou received his Diploma in Electrical and Mechanical Engineering from the National Technical University of Athens in 1982 and his MSc in Computer Science from University College London in 1986. He has since worked in the Computer Science department at UCL mainly as a researcher but also as a lecturer. He is now a Senior Research Scientist and has been leading research efforts in the area of management of broadband networks and services.

David Griffin received the B.Sc. degree in Electronic, Computer and Systems Engineering from Loughborough University, UK in 1988. He joined GEC Plessey Telecommunications Ltd., UK as a Systems Design Engineer working on TMN architectures and ATM traffic experiments. In 1993, Mr. Griffin joined ICS-FORTH as a Research Associate on the EU RACE II ICM project. He joined UCL in 1996 and is currently employed as a Research Fellow working on a number of EU ACTS projects in the area of resource management for TINA systems covering performance, fault, configuration and accounting management. 